\title{
Wave propagation simulation in underground mines by SPECFEM2D
}

\author{
X Wang Laurentian University, Canada \\ M Cai Laurentian University, Canada
}

\begin{abstract}
In burst-prone underground mines, seismic waves generated from a fault slip seismic event may play a critical role in causing relatively large, localised rockburst damage. This study hypothesises that altered wave pattern due to geological structures and mine excavations is one of the important causes of localised rockburst damage resulted from a relatively large fault slip seismic event. The study aims to better understand wave propagation patterns around mine tunnels and to capture peak particle velocity (ppv) accurately for rockburst damage forensic analysis and dynamic support design. For these purposes and as the first step, advanced seismic wave propagation modelling tool SPECFEM2D is used to study complex wave propagation patterns in underground mines.
\end{abstract}

In the present study, particular attention is directed to the influence of different mine excavations and geological structures on wavefield patterns. The simulation results show that the ppv distribution around a tunnel can be altered largely, leading to high and low ppv zones around the tunnel. Moreover, the response of ground motion and wavefields become more complicated as more mine excavations and geological structures are involved; modulation of travel time and long S-coda waves can be observed in the complex waveforms. Using the modelling approach, areas in a mine that may experience high potential of rockburst damage could be identified and correlated to field observation, and mine safety could be improved by implementing dynamic rock ground support in these areas.

\section{Introduction}

As mining occurs increasingly at greater depths, rockburst issue is becoming inevitable due to high in situ stress and complex geological and geometrical conditions in underground hard rock mines. Severe damage to tunnel systems and mining equipment, as well as injuries and fatalities to workers are encountered occasionally due to violent failure of rock mass in underground mines (Ortlepp 1992, 1993; Stacey \& Ortlepp 1994; Kaiser et al. 1996; Lightfoot et al. 1996; Durrheim et al. 1997; Yeryomenko et al. 1999; Cai et al. 2000; Hassani et al. 2001; Wang et al. 2004; Cai \& Champaigne 2009; Cai 2013; Kaiser \& Cai 2013). The rock mass around a tunnel may already reach a state near failure due to mining-induced stress increase, and additional seismic energy input to the rock mass may bring the rock mass to failure. If the failure is unstable, rock ejection may result, causing severe damage. Hence, seismic waves generated from a fault slip event play a critical role in causing large localised damage in underground mines.

Seismic waves emitted from a relatively large fault-slip seismic event can cause localised tunnel damage and this phenomenon can be in part attributed to the complex wave pattern around the underground openings. The propagation patterns of seismic waves are influenced by various factors such as characteristics of the seismic source and the transit medium (Carcione 2001, 2007; Kühn \& Vavryčuk 2013). It is known that medium heterogeneity contributes largely to the variation of wave patterns, which can make the wave patterns very complex. In general, heterogeneities in underground mines are caused by the presence of faults, ore bodies, and different types of rock, mined and backfilled stopes, and tunnel systems. The phenomena of wave reflection, refraction, dispersion, diffraction can be seen when the seismic waves encounter a change of material property in underground mines, and they further complicate the wave propagation pattern. Hence, to conduct rational engineering design such as rock support design, it is important to have a good knowledge of the wave pattern and distribution of peak particle velocity (ppv) near mine openings in the design stage. 
Kaiser et al. (1996) proposed a semi-empirical design scaling law, which has been widely used for ppv estimation in dynamic support design in underground mines. However, the design scaling law cannot consider wave altering factors such as boundary condition and heterogeneity. When analysing rockburst damage and linking the degree of damage to ground motion, actual wave patterns are required. For this purpose, advanced numerical modelling can be employed to estimate the distribution of ground motion around excavations in underground mines and to explain the influence of various factors on the wave patterns.

For wave propagation modelling, it is noticed that research (Faccioli et al. (1997); Komatitsch \& Tromp (1999); Komatitsch et al. (1999); Fichtner (2011) have been conducted in earthquake engineering using the SPECFEM2D/3D codes and the obtained results were in good agreement with the recorded seismograms, however, little work has been conducted using the SPECFEM codes to simulate dynamic wave propagation in underground mines. Because of the merits the SPECFEM codes have (Carrington et al. 2008; Komatitsch et al. 2008; Computational Infrastructure for Geodynamics 2013), it opens the door for seismic wave propagation modelling in mining engineering and would also narrow the gap between seismic wave propagation modelling in earthquake engineering and mining engineering. It is expected that the SPECFEM codes can find their application in underground mining. Hence, this study aims at using this powerful software package to study seismic wave propagation in underground mines.

In this study, the modelling results of wave propagation with typical mine openings and geological structures in 2D models are presented. Wave propagation pattern due to heterogeneity in mines is examined by snapshots of wavefield, synthetic seismograms, and ppv contours around the tunnel.

\section{Brief introduction of SPECFEM for wave propagation modelling}

Different numerical methods, such as finite difference method (FDM) (Moczo et al. 2007a; Moczo et al. 2007b), finite element method (FEM) (Fichtner 2011), pseudo-spectral method (PSM) (Furumura et al. 1998; Wang et al. 2001), spectral-element method (SEM) (Seriani et al. 1995; Faccioli et al. 1997), can be used to solve wave field equations. SEM is a half way between FEM and PSM methods. It can incorporate flexible geometry in FEM and subdivide the computational domain into non-overlapping elements. For excellent reviews of the SEM method, please refer to Komatitsch \& Tromp (1999), Komatitsch et al. (1999), Komatitsch \& Tromp (2002), and Tromp et al. (2008).

SPECFEM2D/3D, based on SEM, are powerful software packages for modelling seismic wave propagation at local or regional scales. They facilitate 2D/3D simulations of acoustic, (an) elastic, and pore-elastic seismic wave propagation (Computational Infrastructure for Geodynamics 2012). The method combines the flexibility of the FEM method with the accuracy of the SEM method. A diagonal mass matrix can be obtained due to the combination of discretisation and integration, which will greatly simplify its algorithm and reduce computing time (Tromp et al. 2008).

For the boundaries of a computation domain, the spectral-element solver in the SPECFEM codes accommodates convolution perfect match layer (PML) absorbing layers and higher-order time schemes (SP Computational Infrastructure for Geodynamics 2012). Convolution or auxiliary differential equation perfectly matched absorbing layers (C-PML or ADE-PML) can also be incorporated in the codes (Komatitsch \& Martin 2007). The 2D package further considers SH (horizontal component of the shear wave) and P-SV (primary wave and vertical component of the shear wave) wave propagation and the solver can run both in serial and in parallel (Computational Infrastructure for Geodynamics 2012, 2013). Effort has been put into parallelising the codes so that the software can be run on supercomputers (Carrington et al. 2008; Komatitsch et al. 2008) and on clusters of GPUs (Komatitsch et al. 2009; Komatitsch et al. 2010; Komatitsch 2011).

Many researchers have proved that these codes have very good accuracy and convergence properties. For example, discussion on high-order numerical methods can be found in Cohen (2002), study of high-order time stepping and high-order finite elements for acoustic or elastic wave propagation can be found in Basabe \& Sen $(2007,2010)$, and research on dispersion analysis can be found in Seriani \& Oliveira (2008). 
In summary, the SPECFEM codes have many merits in the context of seismic wave propagation modelling. It can handle distorted mesh elements accurately, take advanced PML absorbing layers, use high-order spatial and time schemes, possess relatively good convergence, obtain high degree of accuracy, demands less computation power, consider heterogeneous and anisotropic materials, and save time by taking parallel computation.

\section{$3 \quad$ Models and parameters of typical heterogeneities in underground mines}

\section{1 $2 \mathrm{D}$ models with typical heterogeneities in underground mines}

In the SPECFEM2D computation models, heterogeneous medium with different structures in underground mines are considered to examine the influence of typical natural or man-made heterogeneities on wave propagation. The models are shown in Figure 1.

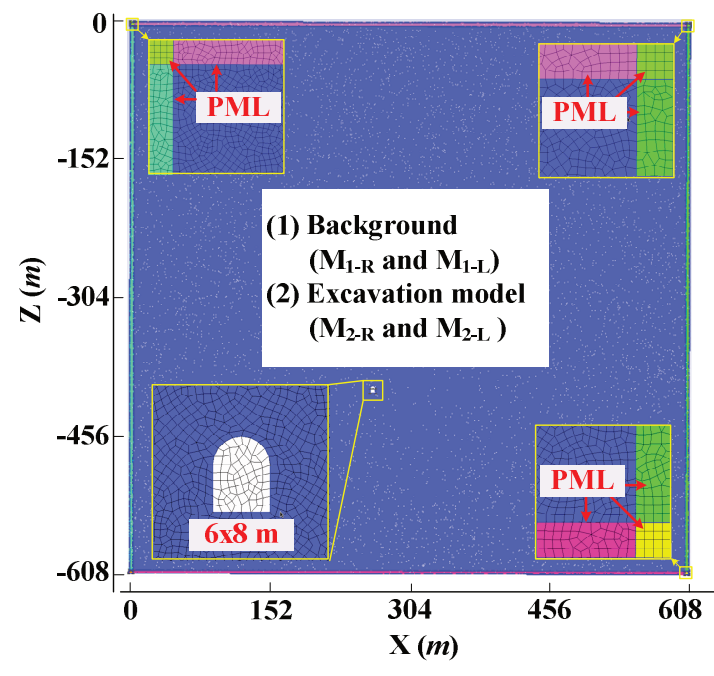

(a)

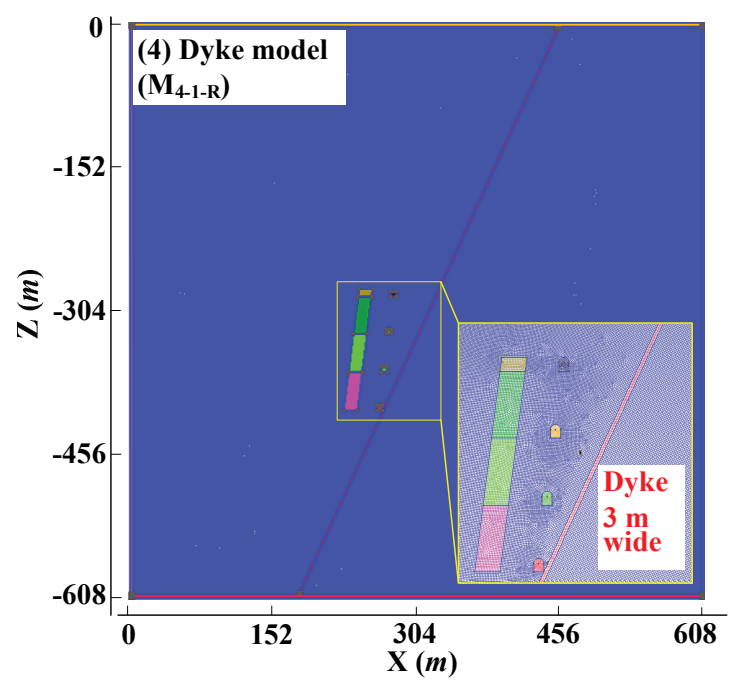

(c)

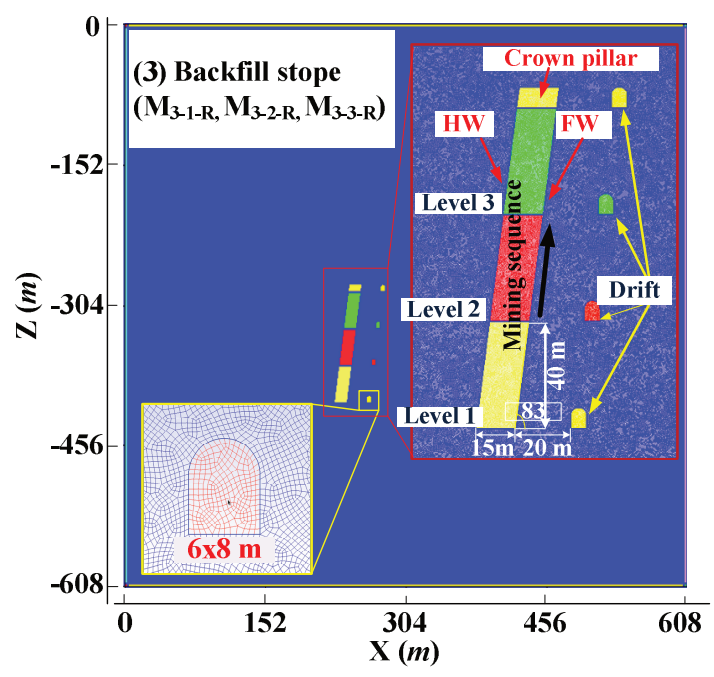

(b)

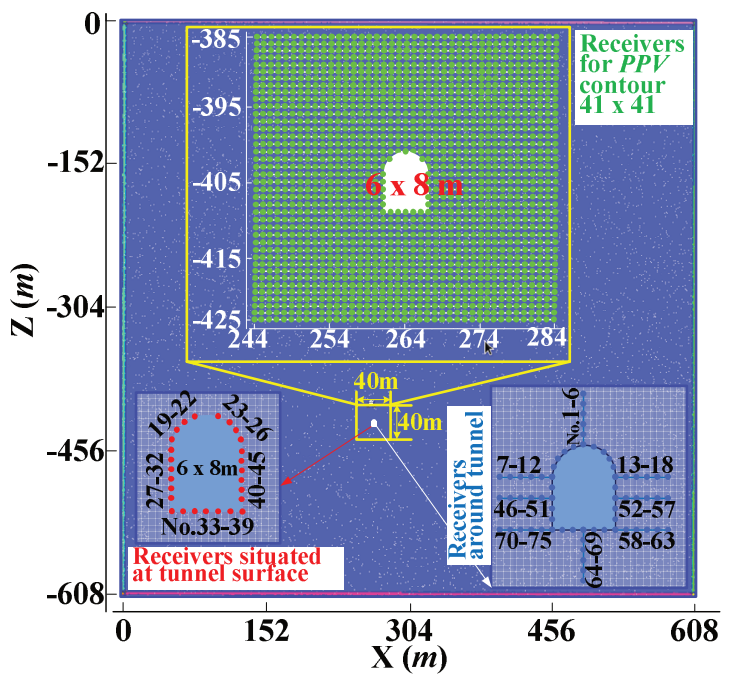

(d)

Figure $12 \mathrm{D}$ models with different heterogeneities whose properties are tabulated in Table 1. (a), (b), and (c) show the background model, the stope model, and the dyke model, respectively; layout of the receivers is shown in (d)

As can be seen from Figure 1, the size of the models is $608 \times 608 \mathrm{~m}^{2}$. The four edges are set as absorbing boundaries and four elements of the PML condition are imposed for each boundary. A $6 \times 8 \mathrm{~m}^{2}$ tunnel is 
located at ( $x=264 \mathrm{~m}, \mathrm{z}=-405 \mathrm{~m}$, the bottom tunnel in the figure). A seismic source is located at a distance from the stopes and tunnels. Several cases are considered: (a) Background model with ( $M_{1-R}$ and $\left.M_{1-L}\right)$ and without a tunnel $\left(M_{2-R}\right.$ and $\left.M_{2-L}\right)$, where $R$ and $L$ in the model name denote that the seismic source is located at the right $(x=550 \mathrm{~m}, z=50 \mathrm{~m})$ and the left $(x=50 \mathrm{~m}, z=-50 \mathrm{~m})$ corners of the model, respectively. The model responses with and without the tunnel can be compared with each other to see the influence of the tunnel on wave propagation pattern. (b) Backfilled stope models $\left(M_{3-1-R}, M_{3-2-R}\right.$, and $M_{3-3-R}$ ), which have multi-level stopes with backfill option (three levels are denoted by 1,2 , and 3 in the middle of the model name). The width and height of the stope are $15 \mathrm{~m}$ and $40 \mathrm{~m}$, respectively, and the dip of the stope is $83^{\circ}$. The distance from the stope to the footwall tunnels is $20 \mathrm{~m}$. To model the influence of different mining stages on wave propagation pattern, bottom-up stoping method is used. (c) Dyke model $\left(\mathrm{M}_{4-1-\mathrm{k}}\right)$, in which a $3.0 \mathrm{~m}$ wide dyke is added. The dyke is inclined such that its distance to the tunnels at different levels is not the same. The dyke is very close to the bottom of the tunnel.

Three different types of receivers are placed at various locations in the model to capture synthetic seismograms and ppv distribution. The layout of the receivers is presented in Figure $1(d) .41$ by 41 receivers are placed regularly in a square around the bottom tunnel to generate ppv contours (black dots in the figure). A set of receivers are placed at the surface of the bottom tunnel for examining the seismic response at the tunnel free surface (dots in Figure 1(d)). In addition, a series of receivers are placed in the vicinity of the tunnel in some lines to identify ppv distributions at different depth from the wall (dots in Figure 1(d)). All these models are created by Gmsh (Geuzaine \& Remacle 2014)and the meshes are discretised using the Delaunay quadrangle algorithm. The grid spacing is about $1 \mathrm{~m}$ and the total number of elements in each model depends on the model configuration.

\subsection{Seismic source parameters and model material properties}

To represent a relatively large fault slip event in underground mines, a normal fault with a dip of $45^{\circ}$ is modelled by the moment tensor point source model. Ricker wavelet source time function with a dominant frequency of $100 \mathrm{~Hz}$ is used. The intensity of the seismic event is assumed with a moment magnitude $M_{\mathrm{w}}=3.0$. Two different positions of the seismic source, top left $(50 \mathrm{~m},-58 \mathrm{~m})$ and top right $(550 \mathrm{~m},-58 \mathrm{~m})$, are considered.

Material properties of ore, backfill, and dyke are listed in Table 1. It should be noted that the shear quality factors $\left(Q_{s}\right)$ for the ore, backfill, and dyke are assumed based on the relative strength of the material when compared with the shear quality factor $\left(Q_{s}=150\right)$ of the background rock material, which was calibrated using the field data based the scaling laws (Kaiser et al. 1996). According to previous research work (Essen et al. 2007; Gharti et al. 2012), the P- and S-wave velocities ( $C_{p}$ and $C_{s}$ ) of openings filled with air are chosen as 600 and $0 \mathrm{~m} / \mathrm{s}$, respectively.

Table 1 Material properties of the heterogeneity models

\begin{tabular}{cccccc}
\hline Variable name & Elastic modulus (GPa) & Poisson's ratio & $\boldsymbol{C}_{\boldsymbol{p}}(\mathrm{m} / \mathrm{s})$ & $\boldsymbol{C}_{\boldsymbol{s}}(\mathrm{m} / \mathrm{s})$ & $\boldsymbol{Q}_{\boldsymbol{s}}$ \\
\hline Background & 58 & 0.24 & 5846 & 3420 & 150 \\
Orebody & 100 & 0.15 & 6372 & 4100 & 300 \\
Backfill & 2.5 & 0.35 & 1830 & 878 & 80 \\
Dyke & 125 & 0.18 & 6726 & 4202 & 400 \\
Tunnel & N/A & N/A & 600 & 0 & 10 \\
\hline
\end{tabular}




\subsection{Influence of a single tunnel on wave propagation}

Figure 2 presents snapshots of the wavefields for the vertical velocity component produced by the seismic event located on the right and left upper corners. For comparison, results from the case without the tunnel are also presented.

As can be seen from Figure 2, the tunnel can alter the velocity of the wavefields significantly. The wavefields of the background models $\left(\mathrm{M}_{1-\mathrm{R}}\right.$ (a) and $\mathrm{M}_{1-\mathrm{L}}$ (c)) show clear P- and S-waves. With the introduction of the horse-shoe tunnel, strong scattering of the S-waves around the tunnel can be observed clearly (at $\mathrm{t}=0.13 \mathrm{~s}$ and $\mathrm{t}=0.15 \mathrm{~s}$ ). The slip direction of the seismic source is defined by a dip of $45^{\circ}$ and when the source is located on the right upper corner, it will have the largest S-wave energy and smallest P-waves energy along a propagation path connected from the source to the tunnel. In this case, the influence of the opening on P-wave propagation is not significant (Figure 2(b), $t=0.08 \mathrm{~s}$ ). Hence, relatively weak P-wave disturbance around the tunnel will result. However, when the seismic source is on the left upper corner, the minimum P-wave energy direction is not in the extension line from the seismic source to the tunnel. As a result, some P-wave disturbances can be seen (Figure $2(d), t=0.08 \mathrm{~s}$ ).
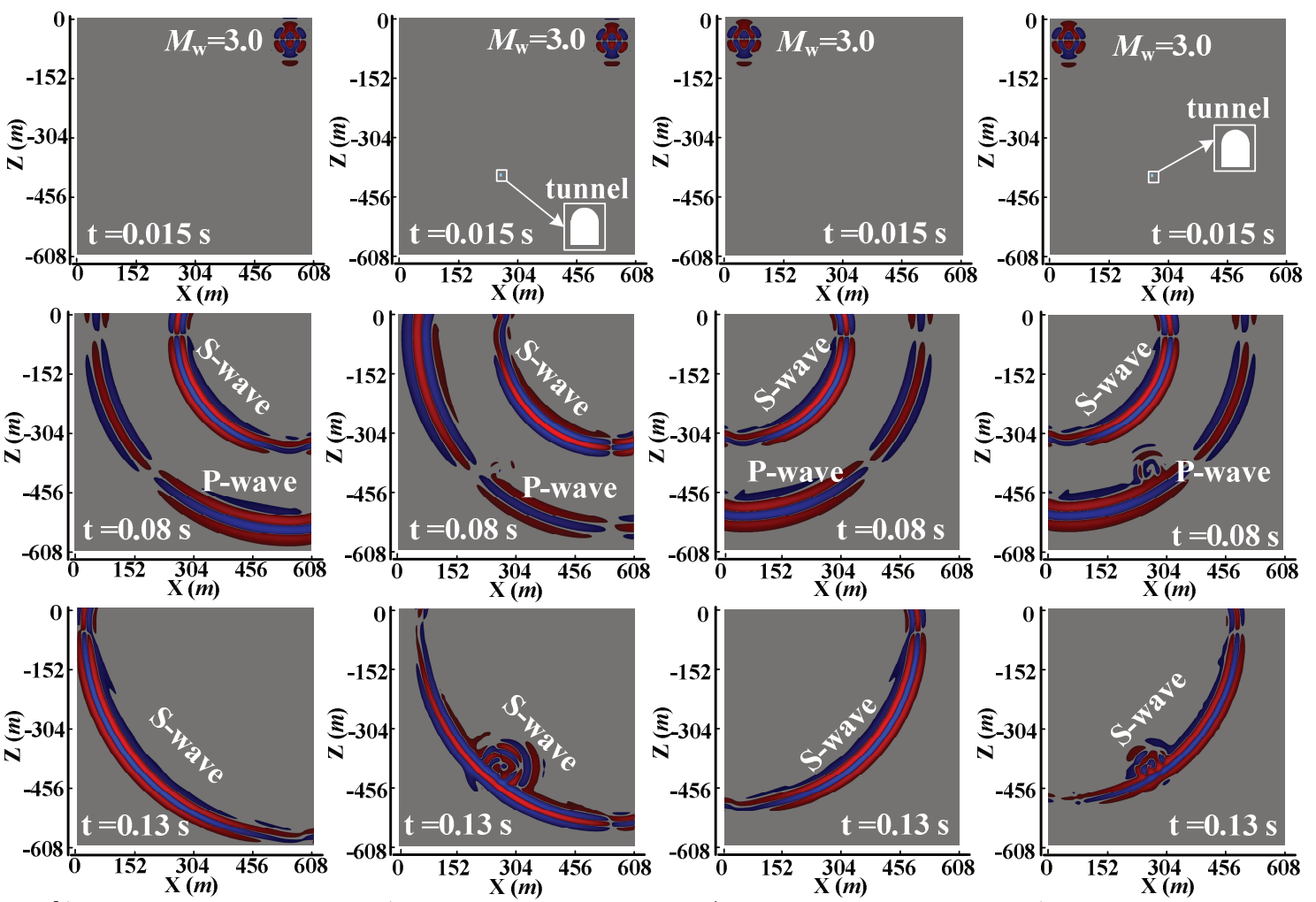

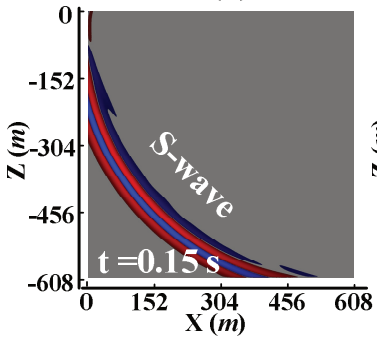

(a) $\mathbf{M}_{1-\mathrm{R}}$

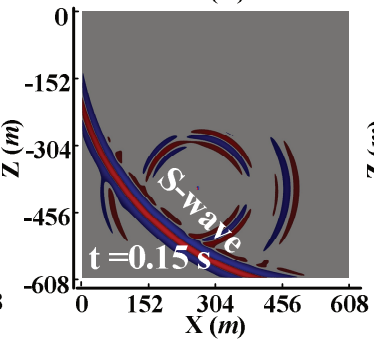

(b) $\mathbf{M}_{2-\mathrm{R}}$

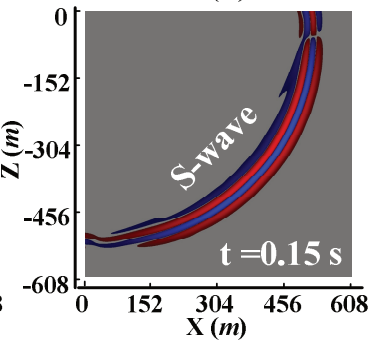

(c) $\mathbf{M}_{1-\mathrm{L}}$

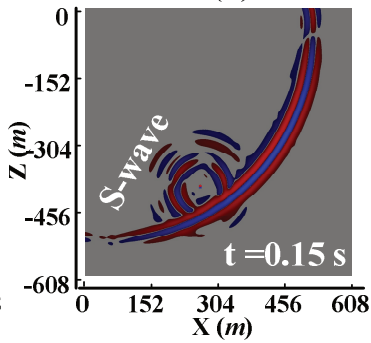

(d) $\mathbf{M}_{2-\mathrm{L}}$

Figure 2 Snapshots of the vertical component of the velocity field at $0.015,0.08,0.13$, and $0.15 \mathrm{~s}$ for four models. The $6 \times 8 \mathrm{~m}^{2}$ horse-shoe shaped tunnel is denoted by blue. Positive and negative values of the velocity components are denoted by light gray (red) and dark black (blue), respectively 
In the $\mathrm{M}_{2-\mathrm{L}}$ and $\mathrm{M}_{2-\mathrm{R}}$ models, although the energy reflected from the P-waves is less intense, strong energy redistribution of the S-waves is clearly noticeable. This means that when the S-waves reach the tunnel, strong scattering waves are excited and dominate the wave propagation after the S-waves pass the tunnel.

To further show the influence of the tunnel on wave propagation, ppv contours, mapped from a $40 \times 40 \mathrm{~m}^{2}$ square area around the opening (Figure 1(d)), are presented in Figure 3 and Figure 4 . The distance between the seismic source and the centre of the tunnel is about 450 and $410 \mathrm{~m}$ for the right and left seismic sources, respectively. According to the design scaling law, the anticipated ppv values at the tunnel centre of the two cases are about 0.1 and $0.11 \mathrm{~m} / \mathrm{s}$. This is based on a point source and isotropic wave propagation assumption. It can be observed clearly that due to the existence of the tunnel the seismic wave energy is redistributed around the tunnel, showing a strong non-uniform ppv distribution around the tunnel with ppv amplified and reduced when comparing with the result from the background ppv. Contrary to the $\mathrm{M}_{1-\mathrm{R}}$ background model, in which ppv decreases gradually as a function of $1 / r$ (where $r$ is the source-target distance), ppv in the excavation model $M_{2-R}$ increases rapidly in areas near the tunnel (Figure 3(a) and (b)).

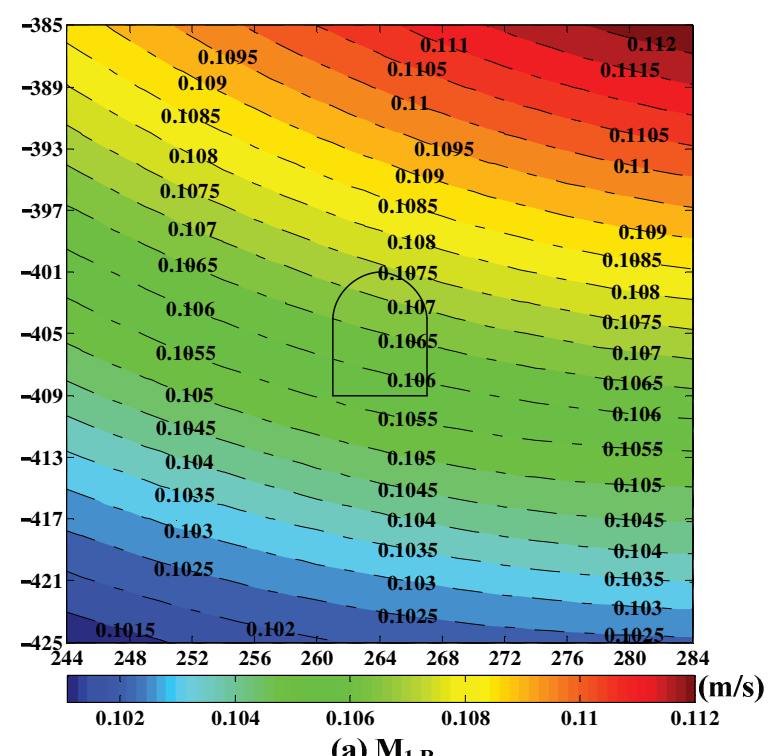

(a) $\mathbf{M}_{1-\mathrm{R}}$

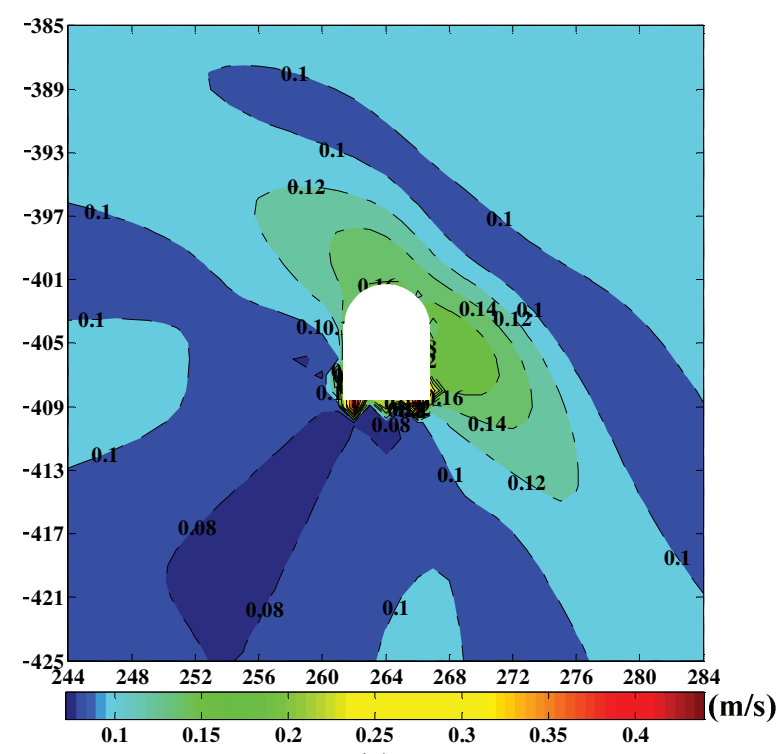

(b) $\mathbf{M}_{2-\mathrm{R}}$

Figure 3 Zoom in ppv contours in (a) the background model; and (b) the model containing a horse-shoe tunnel when the seismic source is placed at the top right at $(x=550 \mathrm{~m}, z=-58 \mathrm{~m})$. Note: the scales of the colour bars are different in these two plots to show clear contours around the tunnel

High ppv zones around the tunnel can be observed in the right hand side wall and on the floor. The maximum ppv is roughly about 4.4 times larger than that in the background model at the floor $(0.44 \mathrm{~m} / \mathrm{s}$ in $M_{2-R}$ and $0.1 \mathrm{~m} / \mathrm{s}$ in $\left.M_{1-R}\right)$. In the meantime, some shield ppv zones can also be identified because of the influence of the tunnel. The right wall and the floor of the tunnel exhibit noticeable high ppv. This may be attributed to the relative locations of the seismic source and the tunnel for the given fault slip direction. This example clearly demonstrates that the actual wave pattern around a tunnel can be very complex and the PPV does not follow the $1 / r$ decay rule anymore. When conducting forensic analysis of rockburst damage, knowing the actual ground motion is important for correlating to actual PPV with ground damage.

Figure 4(a) and (b) show the ppv contours when the seismic source is located at the top left, with the same source parameters and dip (or slip direction). The path from the seismic source to the tunnel is not corresponding to the strongest ground motion. Hence, the $\mathrm{ppv}$ value at the tunnel centre (about $0.0443 \mathrm{~m} / \mathrm{s}$ ) is less than the value from the scaling law (about $0.11 \mathrm{~m} / \mathrm{s}$ ), showing the influence of slip direction on wave pattern. When the tunnel is introduced, the ppv distribution pattern around the tunnel is less altered and basically follows the same pattern as the one in the $M_{1-L}$ model, except some changes near the left side of the roof which is facing the propagation direction of the wavefront. For this model with a 
normal fault source, the maximum direction of P-wave and S-wave are perpendicular and parallel to the fault slip direction, respectively.

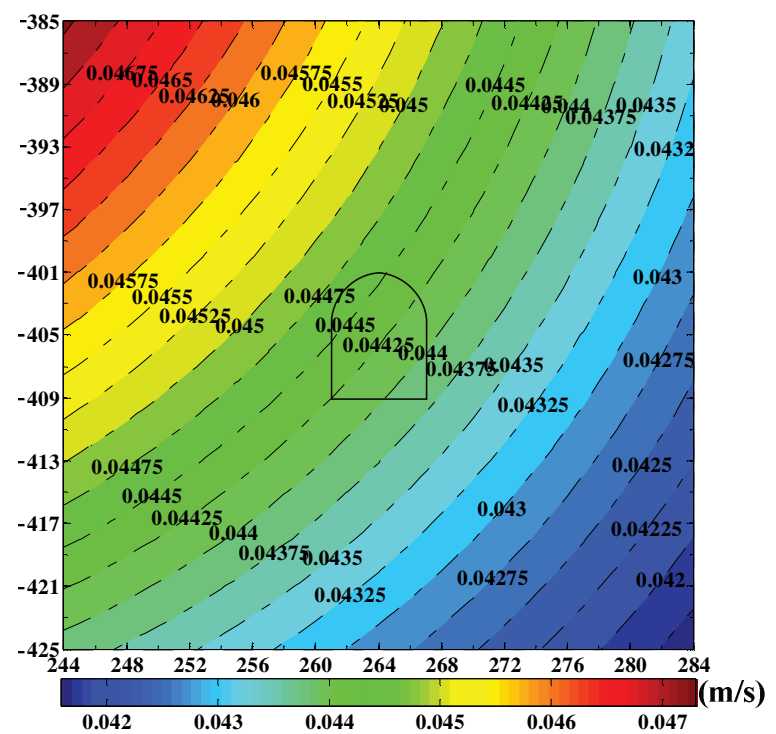

(a) $M_{1-L}$

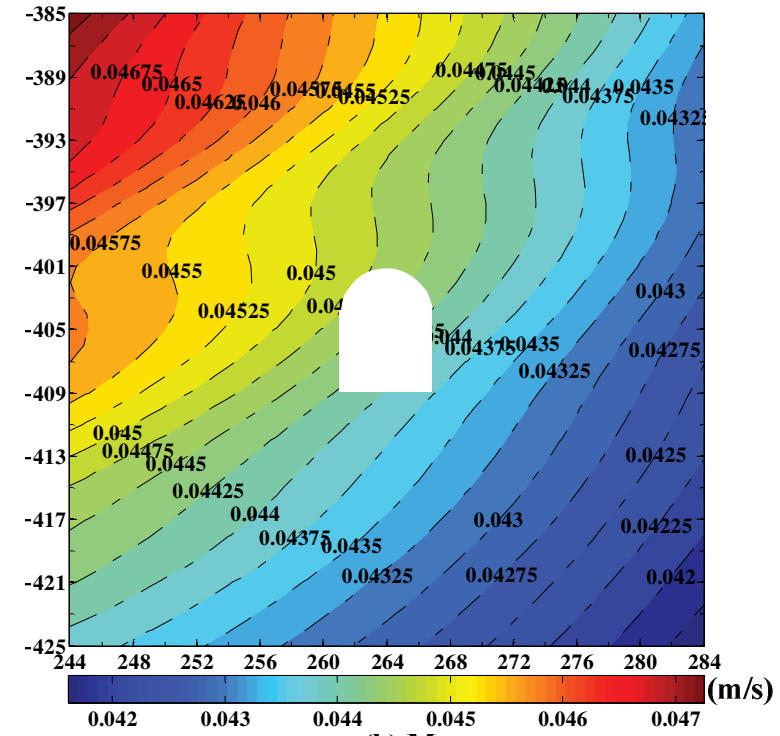

(b) $\mathbf{M}_{2-\mathrm{L}}$

Figure 4 Zoom in ppv contours in (a) the background model and (b) the model containing a horse-shoe tunnel when the seismic source is placed at the top left at $(x=50 \mathrm{~m}, z=-58 \mathrm{~m})$

The ppv from the model without excavation indicates that the ppv estimated from the design scaling law will provide the upper bound of ppv, and both the right and left side source models support this statement. However, the actual ppv near the tunnel boundary can be several times larger than the ppv from the design scaling law if an excavation is present (Figure 3(b)); it can also be smaller than the ppv from the design scaling law due to the influence of the fault slip direction (Figure 4 (b)). One possible reason for the difference is that the data used for deriving the scaling law were not measured directly from tunnel surface. Most field monitoring sensors are located in boreholes sufficiently away from the tunnel surface. Hence, localised amplification effect at tunnel surface is rarely seen in the database. If sensors are placed near or at the tunnel surface, large ground motion due to the geometry effect should be captured. It is clear from Figure 3 and Figure 4 that ppv amplify/shield regions exist around the tunnel and the wave patterns can be altered depending on the location of the seismic source and the source parameters as well as the relative position of the tunnel.

\subsection{Influence of stope and backfill on wave propagation}

In underground mines the mined-out orebody and the backfilled stopes can alter the wave patterns. To examine the influence of different mining stages on wave patterns around a tunnel of interest (the bottom tunnel in Figure 1(b)), models with three levels of stopes are considered. Bottom up mining method is used and backfill of the mined-out stopes is also considered. For example, model $\mathrm{M}_{3-1-\mathrm{R}}$ means that the first stope is excavated and remains open; model $M_{3-2-\mathrm{R}}$ means that stope 1 is backfilled and stope 2 is excavated and remains open; model $M_{3-3-R}$ means that stopes 1 and 2 are backfilled and stope 3 is excavated and remains open. Snapshots of vertical velocity components at different mining stages are presented in Figure 5 at four different times.

Very complex wavefields result from the existence of stopes. Strong scattering waves are observed when the P-waves reach the top of the stope $(t=0.08 \mathrm{~s})$. After the P-waves pass the stopes, $\mathrm{S}$-waves arrive and they interact with the orebody, open stope, and the backfill materials $(t=0.13 \mathrm{~s})$. As time passes, the wavefields become even more complex due to co-existence of multiple reflection and refraction waves. The most notable feature is that complex scattering waves are generated and propagated due to multiple interactions among the host rock, orebody, stopes, backfill, and the tunnel ( $t=0.18 \mathrm{~s})$. The result can be 
used to explain the phenomenon of complex waveforms observed in actual underground mines, suggesting that extremely complex wave patterns are in part due to the complex environment in underground mines and in part due to fault slip that has preferred slip direction.
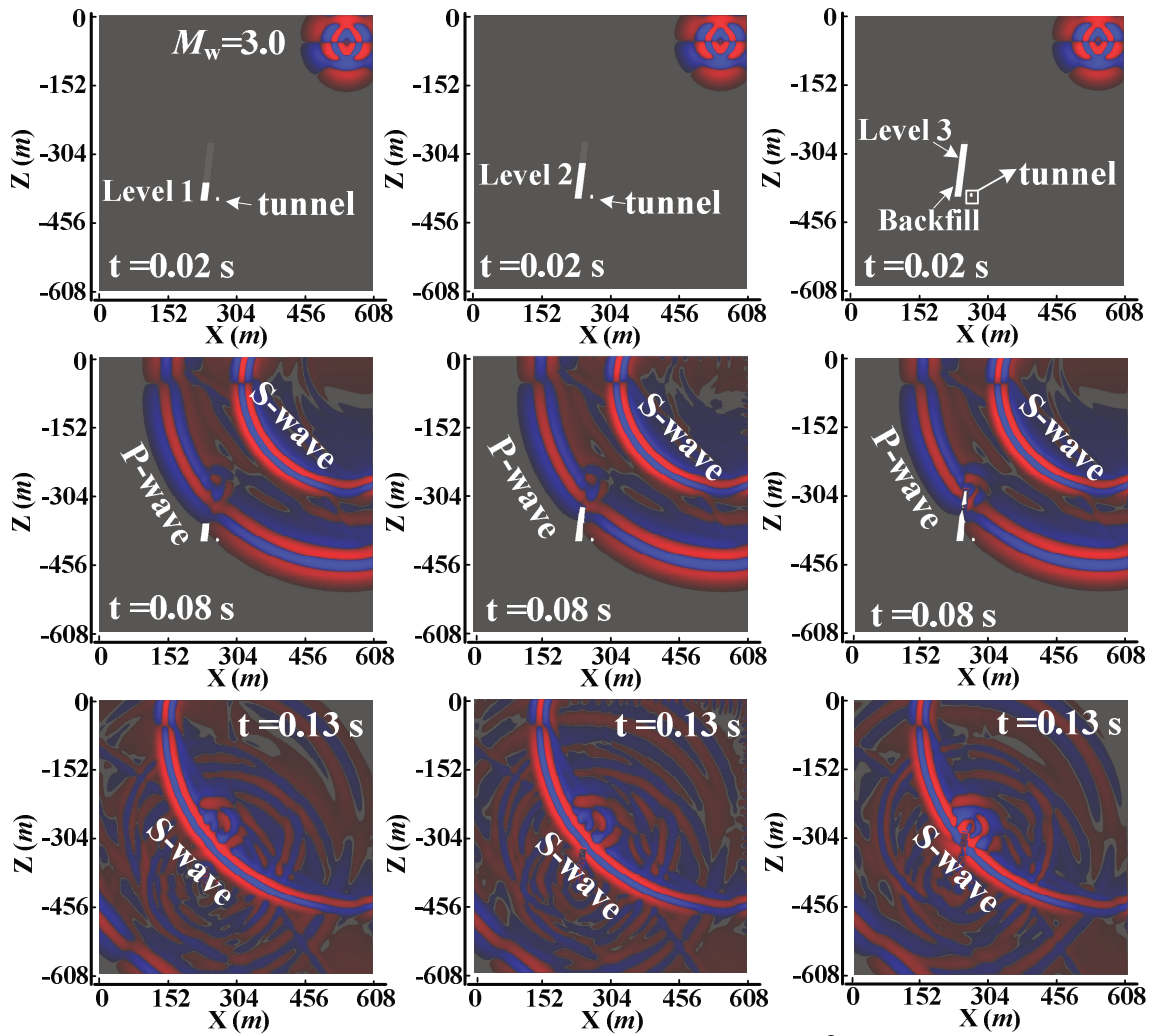

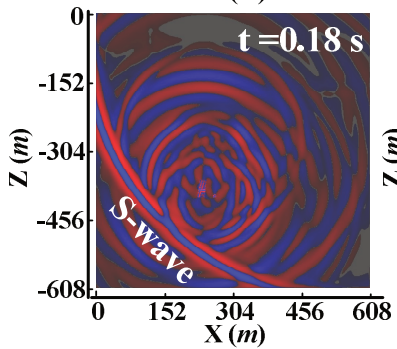

(a) $\mathbf{M}_{3-1-\mathrm{R}}$

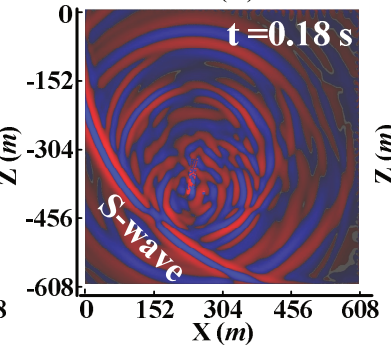

(b) $\mathbf{M}_{3-2-\mathrm{R}}$

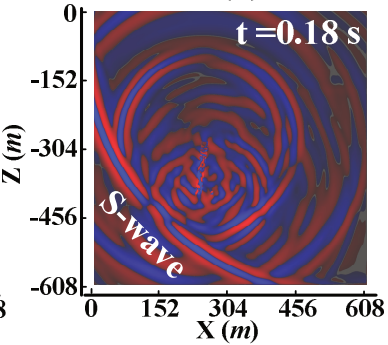

(c) $\mathbf{M}_{3-3-\mathrm{R}}$

Figure 5 Snapshots of the vertical velocity component at $0.02,0.08,0.13$, and $0.18 \mathrm{~s}$ at three different mining stages. The positive and negative values of the vertical velocity components are represented by light gray (red) and dark black (blue), respectively. Upward and downward movements are coloured as light gray (red) and dark black (blue), respectively

ppv contours at different mining stages are presented in Figure 6. There are zones of ppv increase and decrease and in general high ppv values concentrate on the right side of the tunnel, in areas 10 to $20 \mathrm{~m}$ away from the tunnel. However, large ppv variation around the tunnel $(0$ to $10 \mathrm{~m})$ can be found in all cases.

In model $M_{3-1-R}$, ppv is enhanced on both sides of the tunnel walls. The left side of the tunnel will suffer comparably higher seismic shaking because the ppv ranges from 0.125 to $0.32 \mathrm{~m} / \mathrm{s}$ (Figure 6 (b)). This is due to the interaction of multiple reflection waves between the mined-out stope and the tunnel. As expected, higher PPV zones can be found around the mined-out stope (see left top corner of Figure 6 (b)). This result indicates that wave interaction between two openings can increase ground motion.

In the $M_{3-2-\mathrm{r}}$ model, the first stope is backfilled and the second stope is mined out. As the mining front progresses toward to the second stage, the ppv contour (Figure 6 (c)) changes significantly when compared with the contour from the first stage in model $M_{3-1-R}$. The zone around the tunnel with high ppv values 
appears to migrate to the top (roughly in a $6 \mathrm{~m}$ range) and to the right side (roughly in a $10 \mathrm{~m}$ range). This indicates that areas of amplification can be expected largely when stope 2 is mined. Relatively low ppv values near the tunnel surface appear in the $M_{3-2-\mathrm{R}}$ model. The range of ppv value is from 0.12 to $0.26 \mathrm{~m} / \mathrm{s}$, compared with up to $0.3 \mathrm{~m} / \mathrm{s}$ in the $\mathrm{M}_{3-1-\mathrm{R}}$ model.

In the $M_{3-3-R}$ model, the first two stopes are backfilled and the third stope is mined out. The ppv contour changes again in this case (Figure 6(d)). The enhanced/shield ppv zones can be observed clearly in the figure, which are at the right and left parts of the PPV mapping area. Comparably high ppv zones in the vicinity of the tunnel appear on the right hand side floor and walls, with the maximum ppv (about $0.78 \mathrm{~m} / \mathrm{s}$ ) occurring on the floor. Saharan et al. (2006) proposed that severe damage will occur if ppv surpass $0.99 \mathrm{~m} / \mathrm{s}$ and damage is expected when the ppv is larger than $0.6 \mathrm{~m} / \mathrm{s}$. High ppvs have been observed in underground mines. For example, 3 and $2.6 \mathrm{~m} / \mathrm{s}$ of ppv were reported at Tau Tona gold mine and at Mponeng gold mine (Milev et al. 2002). It should be noted that many factors can lead to rock mass damage in underground mines, such as stress condition, rock mass quality, rock support, mine stiffness, etc., and ppv is one of the influence factors.

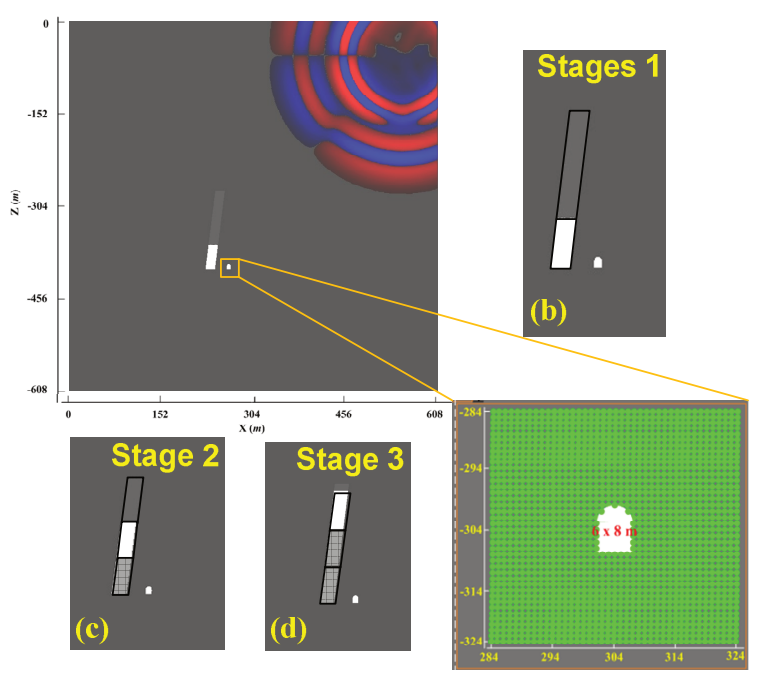

(a)

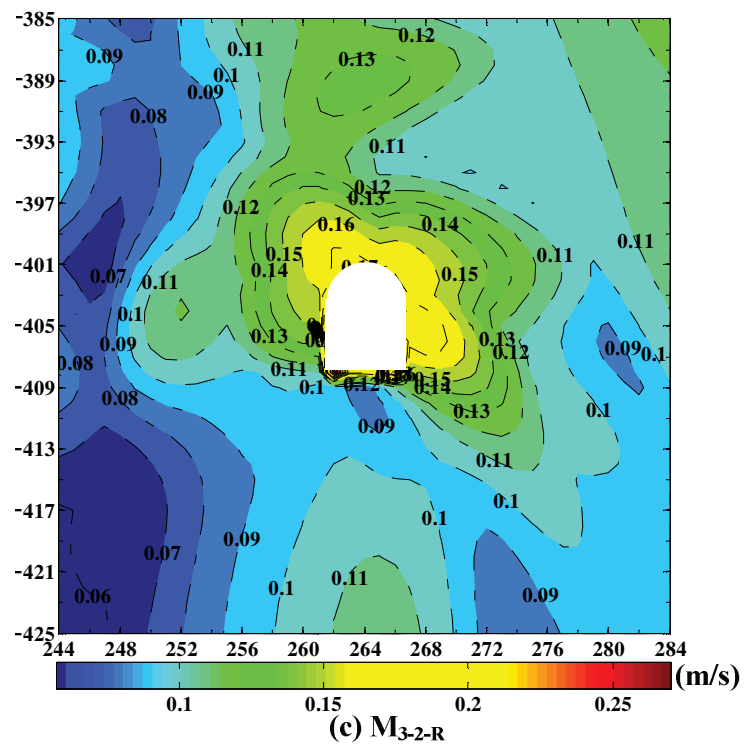

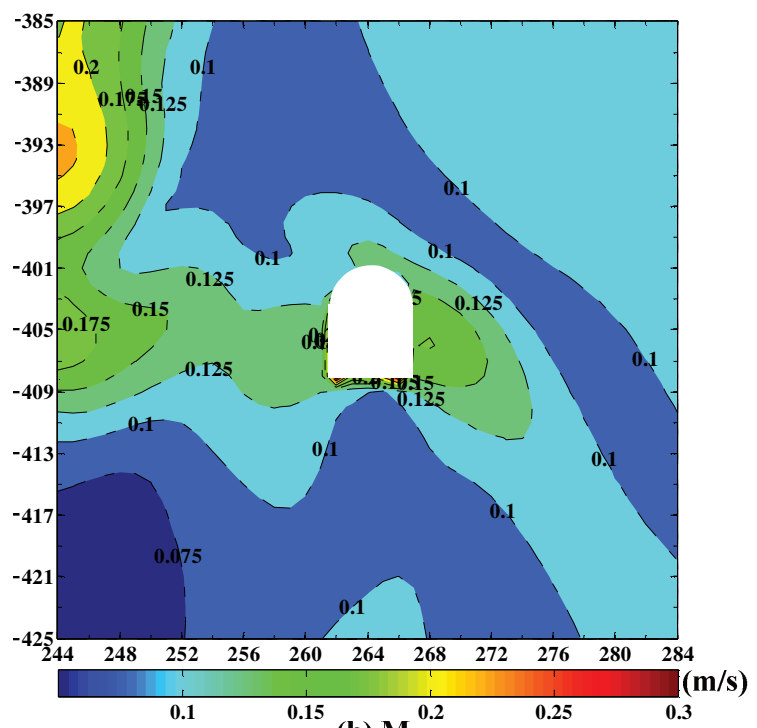

(b) $\mathrm{M}_{3-1-\mathrm{R}}^{\mathrm{0.2}}$

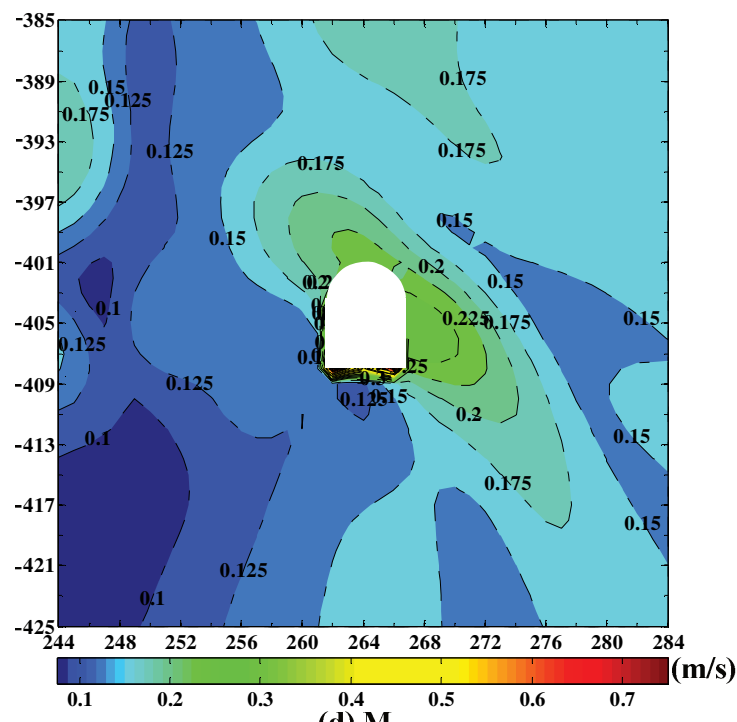

(d) $\mathbf{M}_{3-3-\mathrm{R}}$

Figure $6 \mathrm{ppv}$ around the bottom tunnel at different mining stages. The top left corner shows the mapping range of ppv and three different mining stages. (b), (c), and (d) present ppv contours at the three mining stages. (Note: scales of the colour bars are different to emphasise the ppv distribution around the tunnel) 
The results on the ppv distribution around the tunnel indicate that different mining stages with a system of mined-out, backfilled, and unmined stopes can have a significant influence on the wave patterns. Seismic response around tunnel also shows large ppv variations at different mining stages. Under ideal conditions, the waves can be amplified due to complex wave interaction, leading to very high ppv values which may cause potentially unstable rock failure. High ppv is a major factor that triggers violent rock failure and numerical tools such as SPECFEM2D provide a powerful approach to identify the potential high risk areas.

\subsection{Influence of dyke on wave propagation}

Dykes are common geological structures in underground mines. Problems such as rockburst could occur when mining or tunnelling near/in a dyke. The influence of a dyke on seismic wave patterns is investigated here. For easy comparison, the same model shown in Figure $1(\mathrm{~b})$ is employed by adding a $3 \mathrm{~m}$ wide dyke and the dyke model is shown in Figure 1(c).

Figure 7(a) presents some snapshots of the horizontal velocity component of the dyke model at four different times. In this model simulation, only the first stope is mined. The most notable influence of the dyke is that strong reflection waves will be generated and transmitted for both $\mathrm{P}$ - and S-waves. At the same time, strong scattering waves are also observed when the $P$-waves reach the unmined stopes $(t=0.08 \mathrm{~s})$. After the P-waves pass the stope, the S-waves arrive $(t=0.13 \mathrm{~s})$ and they interact with the orebody and the openings. At a later stage of the simulation, the wavefields become more complex and it is hard to distinguish between reflection and transition waves. The wavefield demonstrates that complex wave patterns can result when there are various structures in a mine.
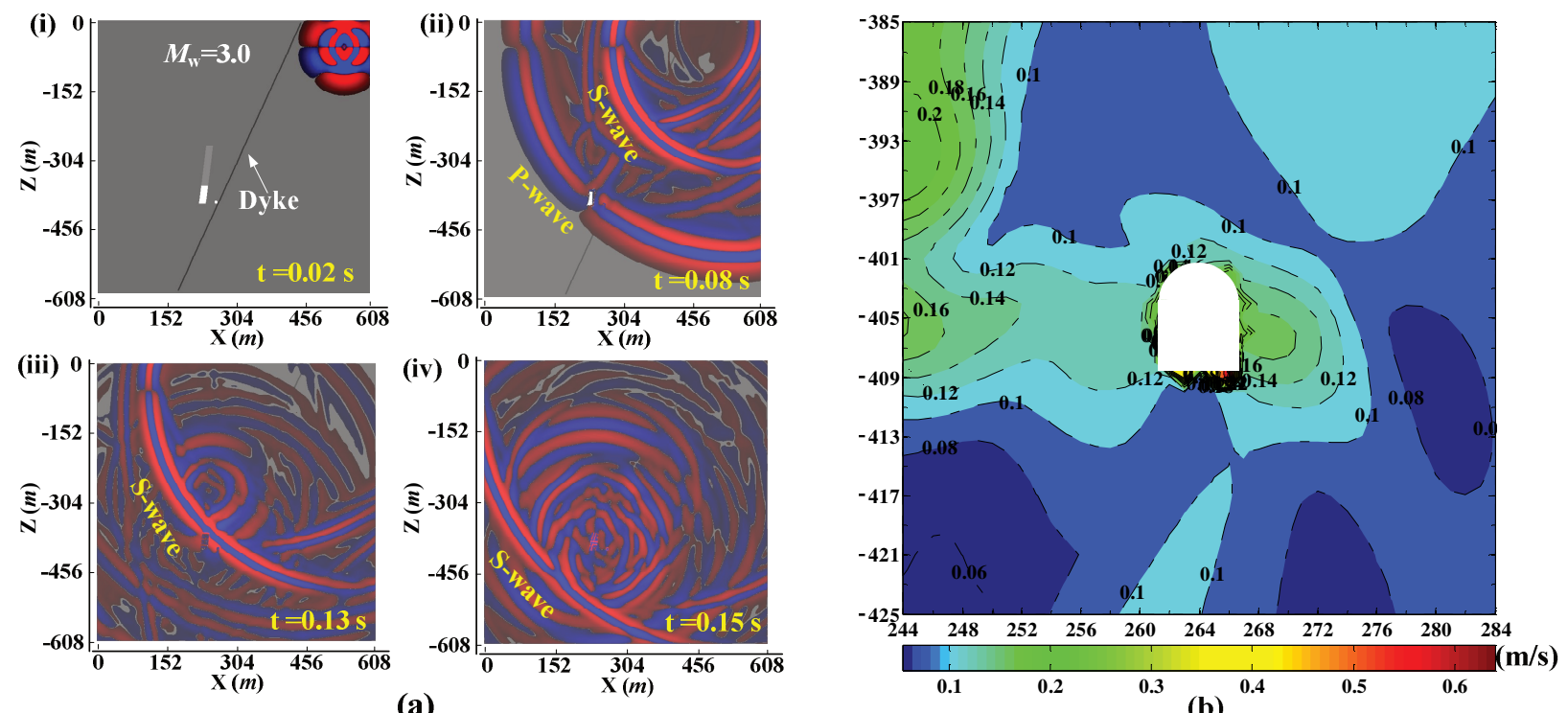

(a)

(b)

Figure 7 (a) snapshots of the horizontal velocity component at $0.02,0.08,0.13$, and $0.15 \mathrm{~s}$ for the dyke model. The positive and negative value is represented by light gray (red) and dark black (blue) (right and left movements are coloured light gray and dark black, respectively); (b) ppv contour around the bottom tunnel. The seismic source is at $(x=550 \mathrm{~m}, z=-58 \mathrm{~m})$ with $M_{W}=3.0$ and dip $=45^{\circ}$

ppv contours for the dyke model are presented in Figure $7(b)$. It can be seen from the figure that there are zones of velocity increase and decrease. The ppv distribution is different from that in Figure 6(b), where there is no dyke. Three shielded and two enhanced zones can be observed in Figure $7(\mathrm{~b})$. On the right side of the figure, there is a large shielded zone about $10 \mathrm{~m}$ away from the tunnel. This may be caused by the fact that the scattering waves, i.e. reflection waves, from the orebody and the stopes are trapped and reflected back to the left part of the dyke. It would be difficult to understand this phenomenon by examining the wavefield snapshot alone, but it becomes obvious by viewing the ppv contour plot. A second shielded zone can be found about $4 \mathrm{~m}$ above the tunnel roof. This may also be caused by the dyke, which can inhibit the waves and cause strong reflection waves for both the P- and S-waves (see wavefields in 
Figure $7(a)$ at $t=0.08,0.3,0.15 \mathrm{~s})$. The third shielded ppv zone is at left bottom side, which may be attributed to the existence of the tunnel. This shielded zone also exists in the model without dyke. One ppv enhanced zone is just around the tunnel and another is near the mined-out stope. The modelling results imply that dykes can influence the wave patterns in underground mines, although the maximum absolute value of ground motion is relatively low in this case $(0.65 \mathrm{~m} / \mathrm{s})$. However, this is more than six times higher than the value predicted by the scaling law, which gives a ppv of $0.1 \mathrm{~m} / \mathrm{s}$ at the tunnel location.

\section{Discussion of the influence of tunnel, stope, and dyke on wave propagation patterns}

The aim of this study is to investigate the influence of heterogeneity on wave pattern and ground motion. Typical heterogeneities in underground mines are considered and modelled. These results show a significant influence of heterogeneity on wave patterns, leading to large variations of ppv around a tunnel. In this section, further analysis and comparison the modelling results from different models are conducted to deepen our understanding of complex wave propagation in underground mines.
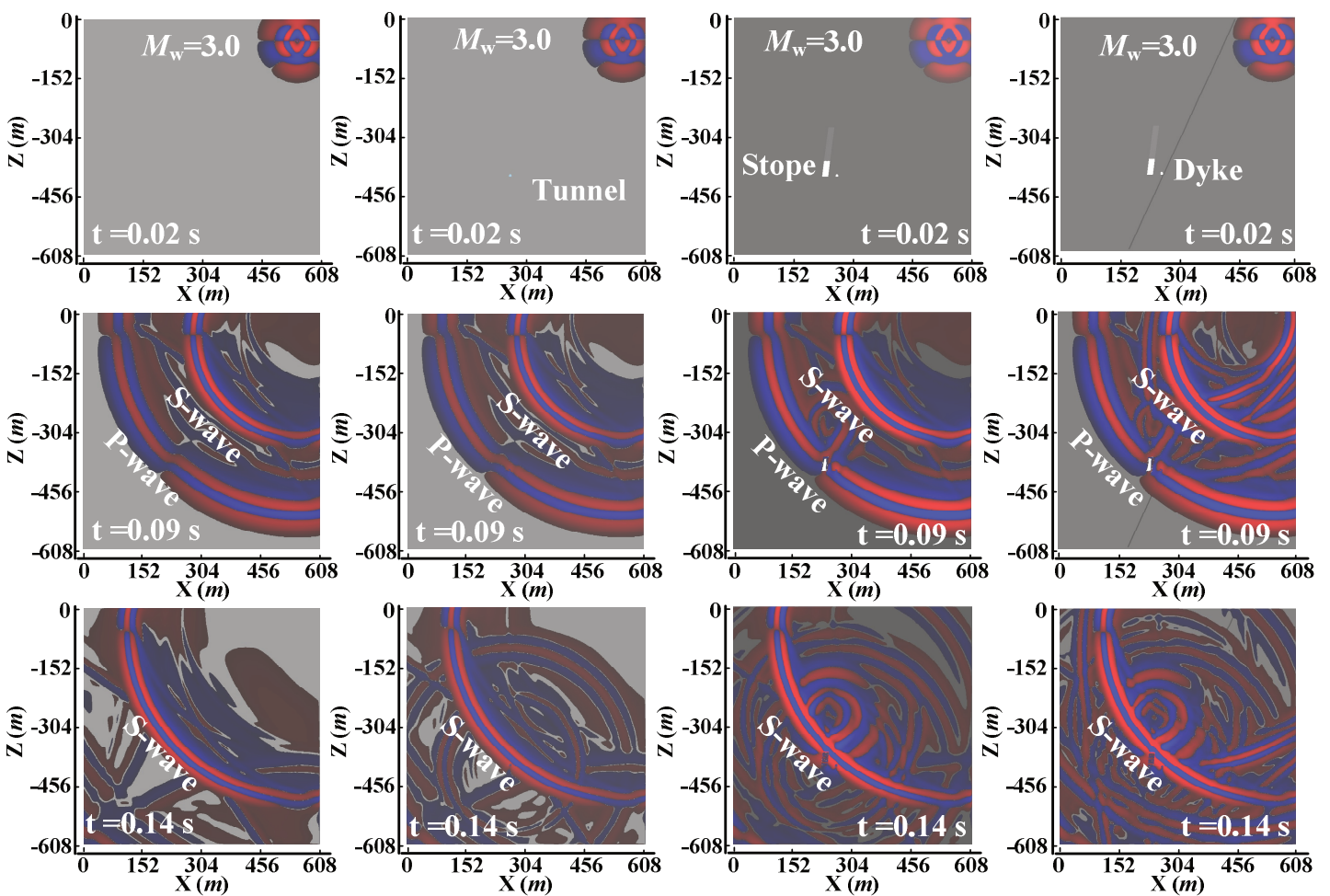

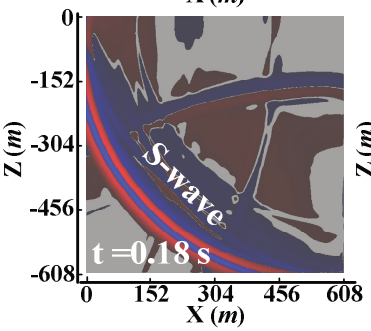

(a) $\mathbf{M}_{1-\mathrm{R}}$

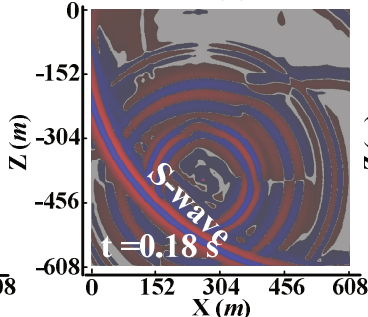

(b) $\mathbf{M}_{2-\mathrm{R}}$

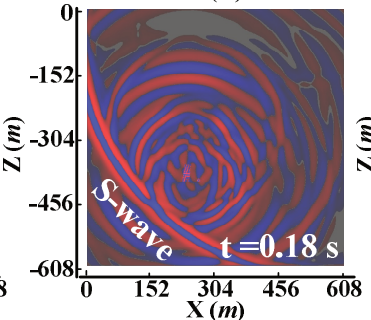

(c) $\mathbf{M}_{3-1-\mathrm{R}}$

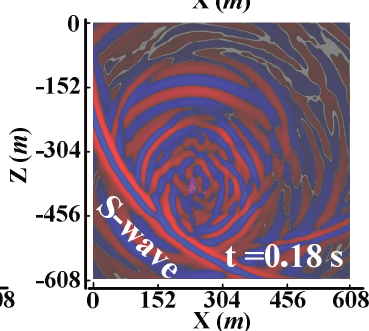

(d) $\mathbf{M}_{4-1-\mathrm{R}}$

Figure 8 Snapshots of the vertical velocity component from 4 different models at $0.02,0.09,0.14$, and $0.18 \mathrm{~s}$. The positive and negative values are represented by light gray (red) and dark black (blue)

Figure 8 presents the snapshots of the vertical velocity component of wavefields for the background model $\left(M_{1-R}\right)$, the single tunnel model $\left(M_{2-k}\right)$, the Stage 1 model $\left(M_{3-1-R}\right)$, and the dyke model $\left(M_{4-1-R}\right)$, at $t=0.02$, $0.09,0.14$, and $0.18 \mathrm{~s}$. To have a have a clear comparison, brightness and contrast of the snapshots havebeen adjusted for each column. Wave appearance behind the direct P- and S- waves are because different display parameters are used. It should be noted that these waves are not boundary reflection waves. It can be seen from the figure that, the complexity of the wavefields increases as more structures 
and excavations are added. Very simple wavefield of direct P- and S-waves are generated and emitted in the background model where the medium is homogeneous (Figure 8(a)). When one excavation is included in the model, strong scattering waves are generated and propagated around the tunnel (Figure 8(b)). When there is stope presence, multiple wave reflection and refraction among these openings can form very complex wavefield (Figure 8(c)). Strong and obvious refection waves can be identified with the presence of the dyke, and the wavefields become extremely complex (Figure 8(d)). The results clearly show that wave patterns can be significantly changed by various structures in underground mines, and amplification or shield of ground motion can be expected in such a case.

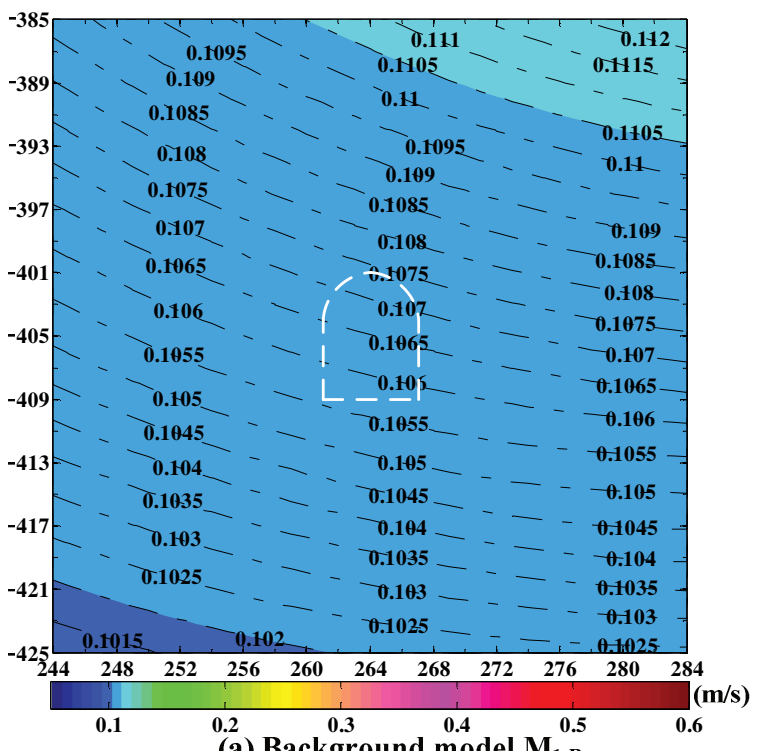

(a) Background model $M_{1-R}$

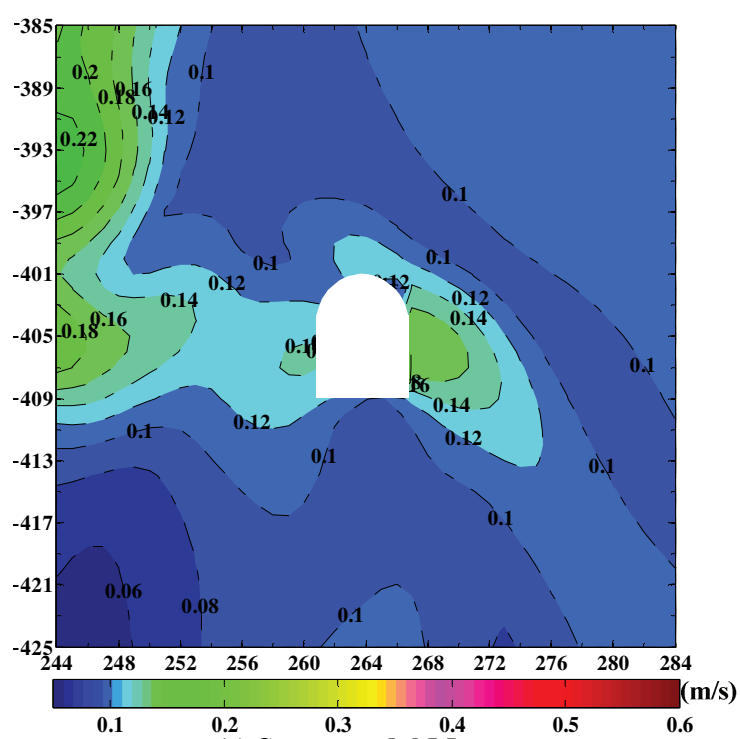

(c) Stope model $M_{3-1-R}$

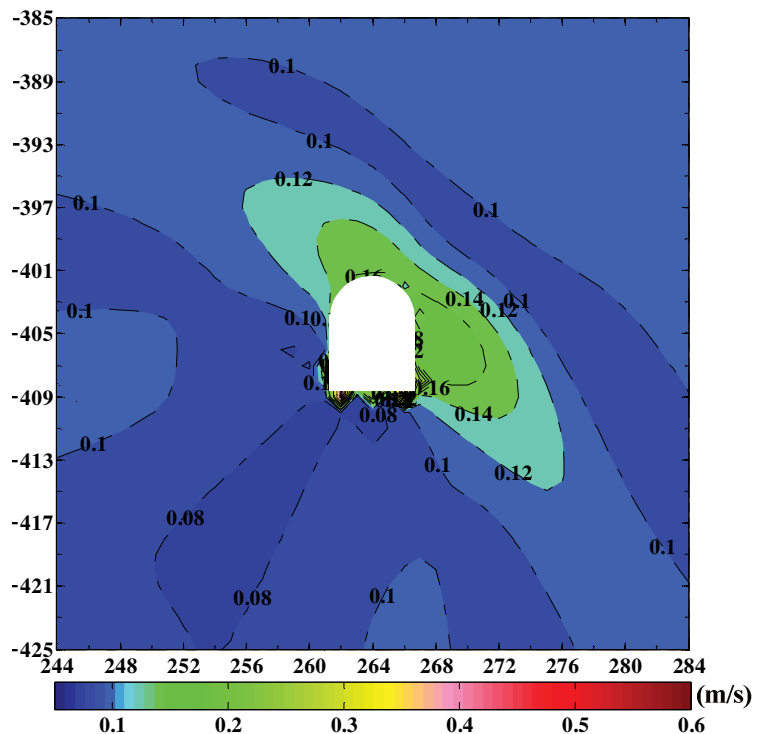

(b) Excavation model $\mathbf{M}_{2-\mathrm{R}}$

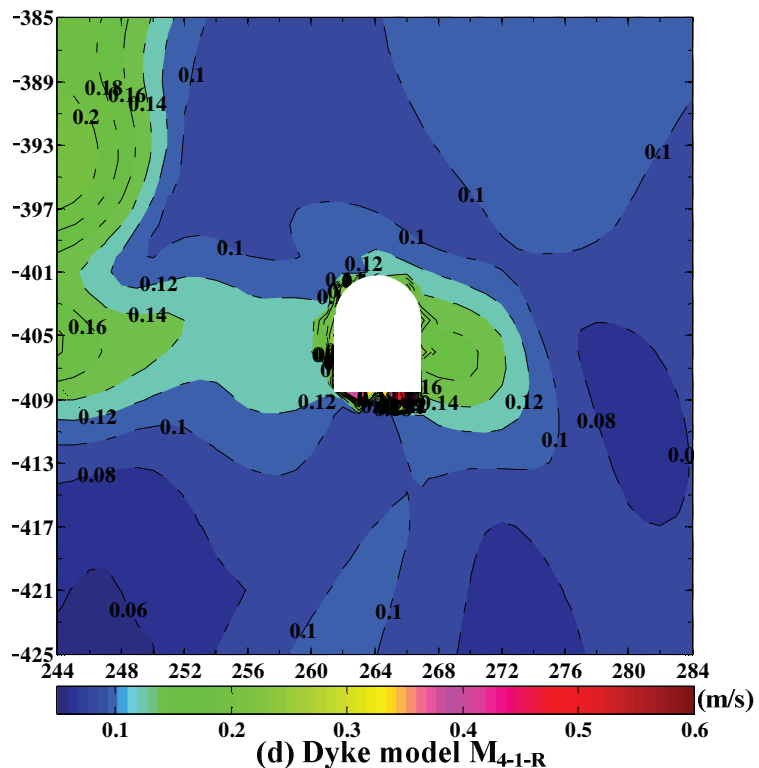

Figure 9 ppv contours around the bottom tunnel for different models. Note: for ease of comparison, the scales of the colour bars are the same, from 0.05 to $0.60 \mathrm{~m} / \mathrm{s}$

Figure 9 compares the ppv contours around the bottom tunnel in the four simulation cases. By comparing ppv distribution with that from homogeneous background model (Figure 9(a)), the influence of mine excavations and geological structures on ppv distribution can be seen. It is seen that ppv increase and decrease zones exist if the medium is not homogenous. The ppv distribution in the background model shows clearly the wave patterns without disturbance, with ppv in the range of 0.102 to $0.112 \mathrm{~m} / \mathrm{s}$ in the mapping area, which is in good agreement with the ppv values estimated from the design scaling law. Once 
a tunnel is considered in the model, the ppv distribution becomes strongly non-uniform (Figure 9(b)), with ppv values ranging from 0.10 to $0.45 \mathrm{~m} / \mathrm{s}$. In other words, ppv can be magnified more than four times due to the tunnel. High ground motion zones are very close to the tunnel floor.

When there is stope presence (Figure 9(c)), the influence of mined-out stope and the tunnel on ppv distribution is evident. The ppv around the tunnel reaches the order of $0.3 \mathrm{~m} / \mathrm{s}$ on both sides of the tunnel, and in a large area. The influence of dyke on wave patterns is interesting (Figure 9(d)). Although the maximum value of ppv is increased to $0.60 \mathrm{~m} / \mathrm{s}$ on the floor, large ppv increase on both sides of the tunnel walls, seen in the model without dyke (Figure 9(c)), is not seen in this case. These results imply that heterogeneity in the form of excavation and geological structures can influence the intensity of the seismic waves and the distribution of ground motions significantly. Both an increase and a decrease of ground motion at a particular location are possible, depending on the types and locations of heterogeneities involved.

Figure 10 presents the synthetic velocity seismograms for four selected receivers of the four models. The seismograms from different models at the same receiver location are compared, and several important observations can be made. For the homogeneous background model, the amplitudes for both the vertical $(0.06 \mathrm{~m} / \mathrm{s})$ and the horizontal velocity components $(0.08 \mathrm{~m} / \mathrm{s})$ are similar at the four receiver locations (light grey solid lines). With the presence of the tunnel $\left(\mathrm{M}_{2-\mathrm{R}}\right)$, the maximum amplitude of the vertical velocity can increase to $0.17 \mathrm{~m} / \mathrm{s}$ and several peaks can be observed for receiver No.28; however, the maximum amplitude at receiver No.41 is only $0.06 \mathrm{~m} / \mathrm{s}$. This is interesting because receiver No.28 has a slightly large source-receiver distance than receiver No.41, but it has a higher amplitude. Similar tendency can be observed for receivers No.38 and No.34 in model $\mathrm{M}_{4-1-\mathrm{R}}$ (the horizontal components are denoted by thick black solid lines).
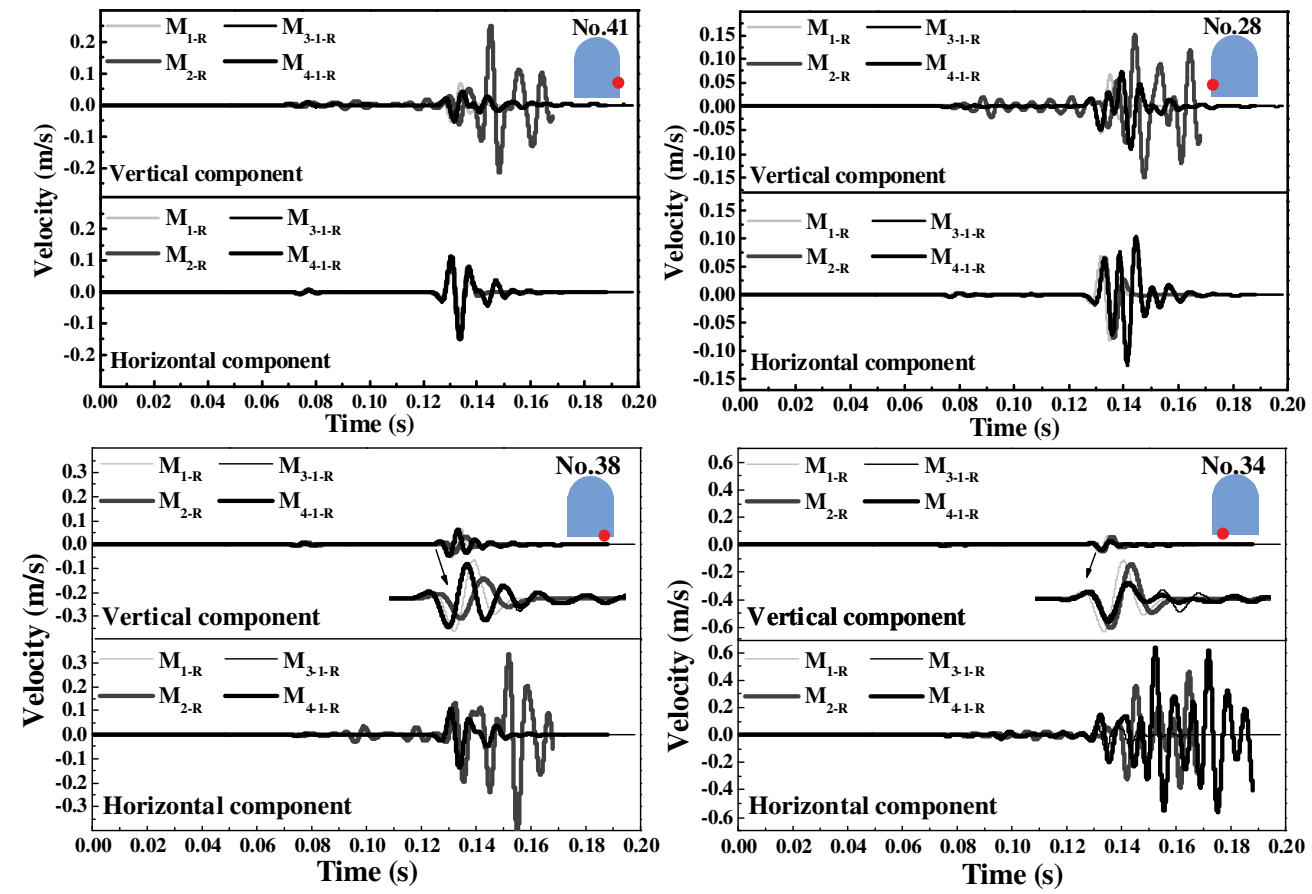

Figure 10 Comparison of synthetic velocity seismograms at receivers No.28, No.34, No.38, and No.41 from different models. Receivers' locations are denoted by the black (red) dots

Furthermore, the maximum amplitudes of the right wall receiver (No.41) in all models are larger than that of the left receiver (No.28) (roughly 1.6 times larger). For the floor receivers No.38 and No.34, the receiver on the left (No.34) experiences stronger ground motions than the receiver on the right (No.38) (roughly 1.8 times larger). Using the seismograms, it is easy to see the contributions of different velocity components to ground motion. For instance, for the receivers located on the tunnel floor, the ground motion comes more 
from the horizontal component than from the vertical component. Of course, the relative contribution is dictated by the influence of excavation and geology as well as seismic source characteristics.

Strong scattering waves can be generated due to heterogeneity and propagate in the medium. Strong coda waves appear as the models become more complex. For instance, noticeable coda waves can be observed from the computed seismogram in the horizontal component at receiver No.34.

Clearly, scattering waves are excited between the direct P- and S-waves, and after direct S-waves pass this receiver (see small oscillations between the $\mathrm{P}$ - and $\mathrm{S}$-waves, and large oscillations after the $\mathrm{S}$-waves in the seismograms). In addition, several large repeated wave peaks after the direct S-waves can be found once the tunnel is added (see the solid thick grey line for receiver No.34 for model $\mathrm{M}_{2-\mathrm{R}}$ ). Similarly, strong coda waves can be found for this receiver (the solid thick grey line) in the dyke model $\left(\mathrm{M}_{4-1-\mathrm{R}}\right)$ and multiple wave peaks can be picked in the seismograms. This indicates that the maximum ground motion may not be directly caused by the direct S-waves; it can be caused by oscillation and shake due to the complex coda waves, which may cause repeated loading to the rock mass, leading to rock damage or even rockburst in underground mines.

P-waves seem to have little impact on seismic response in all cases. Strong seismic response, which results from the S-waves can be observed at all the receivers in all models. This observation further indicates that tunnel damage can be caused by shear waves and coda waves generated from the shear waves. In addition, arrival time delay and advance due to the influence of different structures can be seen in the results. Comparably large arrival time delay can be observed as more heterogeneity is included in the models.

\section{Conclusions}

The modelling results demonstrate the influence of geological and mine structures on seismic wave propagation. Wave propagation patterns around a tunnel can be altered due to various heterogeneities in underground mines. In particular, the following conclusions are obtained.

- Strong scattering waves can be excited and they dominate the wavefields after the direct waves pass the excavation tunnel. Because the existence of the tunnel, the seismic wave energy is redistributed around the tunnel, leading to non-uniform distribution of ppv. In addition, amplification and shield ppv zones can be found around the tunnel.

- The different mining stages influence the local wave pattern. Because of the increase and decrease effect caused by the mined-out area and backfilling, seismic response around the tunnel shows large variations at different mining stages. When the incoming seismic waves reach the tunnel, higher ppv values can be caused by the complex wave interaction. The resulting high ppv may trigger and cause unstable rock failure.

- The most notable feature of the influence of dyke on seismic wave propagation is the generation of strong reflection waves for both P- and S-waves. The wavefield and wave patterns can become very complex when there are various structures. In addition, multiple openings, in combination with the geological structure, can alter the wave patterns and absorb seismic energy, leading to the shield effect in some areas around the tunnel.

- Scatter waves and strong coda waves are the result of the influence of heterogeneities on P-and S-wave propagation.

In summary, ppv amplification and shield effect can occur in underground mines, particularly around excavation boundaries where mining-induced stresses are high. As a result, rockburst damage may be triggered in underground mines at locations where a combination of high stress and high ppv is materialised. The numerical modelling wave propagation is important for conducting forensic analysis of rockburst damage and for developing new design rationale for improved rock support design. 


\section{Acknowledgement}

This work has been supported by the Ontario Research Fund (for a project entitled 'Smart Underground Monitoring and Integrated Technologies (SUMIT) for Deep Mining'), Vale, Rio Tinto, and Glencore. We gratefully thank the CIG (Computational Infrastructure for Geodynamics) for providing the open-source software package SPECFEM2D.

\section{References}

Basabe, JDD \& Sen, MK 2007, 'Grid dispersion and stability criteria of some common finite-element methods for acoustic and elastic wave equations', Geophysics, vol. 72, no. 6, pp. T81-T95.

Basabe, JDD \& Sen, MK 2010, 'Stability of the high-order finite elements for acoustic or elastic wave propagation with high-order time stepping', Geophysical Journal International, vol. 181, no. 1, pp. 577-590.

Cai, M 2013, 'Principles of rock support in burst-prone ground', Tunnelling and Underground Space Technology, vol. 36, pp. 46-56.

Cai, M \& Champaigne, D 2009, 'The art of rock support in burst-prone ground', in CA Tang (ed.), Proceedings of the Seventh International Symposium on Rockburst and Seismicity in Mines: RaSiM7, Rinton Press, Paramus, pp. 33-46.

Cai, M, Kaiser, PK, Uno, H \& Tasaka, Y 2000, 'Comparative study of rock support system design practice for large-scale underground excavations', Proceedings of the 4th North American Rock Mechanics Symposium, A.A. Balkema, Rotterdam, pp. 1027-1034.

Carcione, JM 2001, Wave Fields in Real Media: Anisotropic, Anelastic and Porous Media, Elsevier Science, Amsterdam.

Carcione, JM 2007, Wave Fields in Real Media: Theory and Numerical Simulation of Wave Propagation in Anisotropic, Anelastic, Porous and Electromagnetic Media, Elsevier Science, Amsterdam.

Carrington, L, Komatitsch, D, Laurenzano, M, Tikir, M, Michea, D, Go, NL, Snavely, A \& Tromp, J 2008, 'High-frequency simulations of global seismic wave propagation using SPECFEM3D_GLOBE on 62 thousand processor cores', Proceedings of the ACM/IEEE Supercomputing SC'2008 Conference, pp. 1-11.

Cohen, G 2002, Higher-order Numerical Methods for Transient Wave Equations, Springer, Berlin.

Durrheim, RJ, Handley, MF, Haile, A, Roberts, MKC \& Ortlepp, WD 1997, 'Rockburst damage to tunnels in a deep South African gold mine caused by a $M=3.6$ seismic event', Rockbursts and Seismicity in Mines, pp. 223-226.

Essen, K, Bohlen, T, Friederich, W \& Meier, T 2007, 'Modelling of Rayleigh-type seam waves in disturbed coal seams and around a coal mine roadway', Geophysical Journal International, vol. 170, pp. 511-526.

Faccioli, E, Maggio, F, Paolucci, R \& Quarteroni, A 1997, '2D and 3D elastic wave propagation by a pseudo-spectral domain decomposition method', Journal of Seismology, vol. 1, no. 3, pp. 237-251.

Fichtner, A 2011, Full Seismic Waveform Modelling and Inversion, Springer, Berlin.

Furumura, T, Kennett, BLN \& Furumura, M 1998, 'Seismic wavefield calculation for laterally heterogeneous whole earth models using the pseudo spectral method', Geophysical Journal International, vol. 135, no. 3, pp. 845-860.

Geuzaine, C \& Remacle, J-F 2014, Gmsh, http://geuz.org/gmsh/

Gharti, HN, Oye, V, Komatitsch, D \& Tromp, J 2012, 'Simulation of multistage excavation based on a 3D spectral-element method', Computers \& Structures, vol. 100, no. 2012, pp. 54-69.

Hassani, F, Ouellet, J \& Servant, S 2001, 'In situ measurements in a paste backfill: Backfill and rock mass response in the context of rockburst', Proceedings of the Seventeenth International Mining Congress and Exhibition of Turkey, pp. 165-175.

Kaiser, PK \& Cai, M 2013, 'Critical review of design principles for rock support in burstprone ground - time to rethink!', in Y Potvin \& B Brady (eds), Proceedings of the Seventh International Symposium on Ground Support in Mining and Underground Construction, Australian Centre for Geomechanics, Perth, pp. 3-38.

Kaiser, PK, Mccreath, DR \& Tannant, DD 1996, Rockburst Support Handbook, Geomechanics Research Centre, Sudbury.

Komatitsch, D 2011, 'Fluid-solid coupling on a cluster of GPU graphics cards for seismic wave propagation', Comptes Rendus Mecanique, vol. 339, no. 2-3, pp. 125-135.

Komatitsch, D, Erlebacher, G, Goddeke, D \& Michea, D 2010, 'High-order finite-element seismic wave propagation modeling with MPI on a large GPU cluster', Journal of Computational Physics, vol. 229, no. 20, pp. 7692-7714.

Komatitsch, D, Labarta, D \& Michea, D 2008, 'A simulation of seismic wave propagation at high resolution in the inner core of the Earth on 2166 processors of MareNostrum', Lecture Notes in Computer Science, vol. 5336, pp. 364-377.

Komatitsch, D \& Martin, R 2007, 'An unsplit convolutional perfectly matched layer improved at grazing incidence for the seismic wave equation', Geophysics, vol. 72, no. 5, pp. Sm155-Sm167.

Komatitsch, D, Michea, D \& Erlebacher, G 2009, 'Porting a high-order finite-element earthquake modeling application to NVIDIA graphics cards using CUDA', Journal of Parallel and Distributed Computing, vol. 69, no. 5, pp. 451-460.

Komatitsch, D \& Tromp, J 1999, 'Introduction to the spectral element method for three-dimensional seismic wave propagation', Geophysical Journal International, vol. 139, no. 3, pp. 806-822.

Komatitsch, D \& Tromp, J 2002, 'Spectral-element simulations of global seismic wave propagation - I. Validation', Geophysical Journal International, vol. 149, no. 2, pp. 390-412.

Komatitsch, D, Vilotte, JP, Vai, R, Castillo-Covarrubias, JM \& Sanchez-Sesma, FJ 1999, 'The spectral element method for elastic wave equations - Application to 2-D and 3-D seismic problems', International Journal for Numerical Methods in Engineering, vol. 45, no. 9, pp. 1139-1164.

Kühn, D \& Vavryčuk, V 2012, 'Determination of full moment tensors of microseismic events in a very heterogeneous mining environment', Tectonophysics, vol. 589, pp. 32-43. 
Lightfoot, N, Goldbach, OD, Kullmann, DH \& Toper, AZ 1996, 'Rockburst control in the South African deep level gold mining industry', Rock Mechanics Tools and Techniques, vol. 1 and 2, pp. 295-303.

Computational Infrastructure for Geodynamics 2012, SPECFEM2D, version 7.0, http://geodynamics.org/cig/software/specfem2d/

Computational Infrastructure for Geodynamics 2013, SPECFEM3D Cartesian, version 2.1, http://geodynamics.org/cig/software /specfem3d/

Milev, AM, Spottiswoode, SM, Noble, BR, Linzer, LM, Zyl, MV, Daehnke, A \& Acheampong, E 2002, The meaningful use of peak particle velocities at excavation surfaces for the optimisation of the rockburst criteria for tunnels and stopes, SIMRAC Final Project Report GAP 709, Council for Scientific and Industrial Research, Pretoria.

Moczo, P, Kristek, J, Galis, M, Pazak, P \& Balazovjech, M 2007a, 'The finite-difference and finite-element modeling of seismic wave propagation and earthquake motion', Acta Physica Slovaca, vol. 57, no. 2, pp. 177-406.

Moczo, P, Robertsson, JOA \& Eisner, L 2007b, 'The finite-difference time-domain method for modeling of seismic wave propagation', Advances in Geophysics, vol. 48, pp. 421-516.

Ortlepp, WD 1992, 'The Design of Support for the Containment of Rockburst Damage in Tunnels - an Engineering Approach', Rock Support in Mining and Underground Construction, pp. 593-609.

Ortlepp, WD 1993, 'High Ground Displacement Velocities Associated with Rockburst Damage', Rockbursts and Seismicity in Mines 93, pp. 101-106.

Saharan, MR, Mitri, HS \& Jethwa, JL 2006, 'Rock fracturing by explosive energy: review of state-of-the-art', Fragblast, vol. 10, no. 1-2, pp. 61-81.

Seriani, G \& Oliveira, SP 2008, 'Dispersion analysis of spectral-element methods for elastic wave propagation', Wave Motion, vol. 45 , no. 6 , pp. $729-744$.

Seriani, G, Priolo, E \& Pregarz, A 1995, 'Modelling Waves in Anisotropic Media by a Spectral Element Method', in G Cohen (ed.), Proceedings of Third International Conference on Mathematical and Numerical Aspects of Wave Propagation, Society for Industrial and Applied Mathematics, Philadelphia, pp. 289-298.

Stacey, TR \& Ortlepp, WD 1994, 'Rockburst Mechanisms and Tunnel Support in Rockburst Conditions', Geomechanics 93, pp. 39-46.

Tromp, J, Komatitsch, D \& Liu, QY 2008, 'Spectral-element and adjoin methods in seismology', Communications in Computational Physics, vol. 3, no. 1, pp. 1-32.

Wang, XB, Yang, XB, Zhang, ZH \& Pan, YS 2004, 'Dynamic analysis of fault rockburst based on gradient-dependent plasticity and energy criterion', Journal of University of Science and Technology Beijing, vol. 11, no. 1, pp. 5-9.

Wang, YB, Takenaka, H \& Furumura, T 2001, 'Modelling seismic wave propagation in a two-dimensional cylindrical whole-earth model using the pseudo spectral method', Geophysical Journal International, vol. 145, no. 3, pp. 689-708.

Yeryomenko, AA, Gaidin, AP, Vaganova, VA \& Yeryomenko, VA 1999, 'Rockburst-hazard criterion of rock mass', Journal of Mining Science, vol. 35, no. 6, pp. 598-601. 